\title{
Giffen paradoxes in quantum market games
}

\author{
Jan Sładkowski \\ Institute of Physics, University of Silesia, \\ Uniwersytecka 4, Pl 40007 Katowice, Poland \\ e-mail: sladk@us.edu.pl
}

\begin{abstract}
Recent development in quantum computation and quantum information theory allows to extend the scope of game theory for the quantum world. The paper presents the history and basic ideas of quantum game theory. Description of Giffen paradoxes in this new formalism is discussed.

PACS numbers: 02.50.Le, 03.67.-a, 03.65.Bz
\end{abstract}

Keywords: quantum games, quantum strategies, econophysics, financial markets

\section{Motivation}

Attention to the very physical aspects of information characterizes the recent research in quantum computation, quantum cryptography and quantum communication. In most of the analysed cases quantum description of the system provides advantages over the classical situation. The flagships of quantum 
information are: famous Shor's polynomial time quantum algorithm for factoring [1], Simon's quantum algorithm to identify the period of a function chosen by an oracle (more efficient than any deterministic or probabilistic algorithm) [2] and the quantum protocols for key distribution, devised by Wiener, Bennett and Brassard, and Ekert (qualitatively more secure against eavesdropping than any classical cryptographic system) [3, 4].

Game theory, the study of (rational) decision making in conflict situation, seems to ask for a quantum version. Games against nature, originally studied by Milnor [6], include those for which nature is quantum mechanical. Many of quantum information problems have game-theoretic counterparts. Finally, von Neumann is one of the founders of both modern game theory [7] and quantum theory. Classical strategies can be pure or mixed: why cannot they be entangled or interfere with each other? Can quantum strategies be more successful than classical ones? Are they of any practical value?

\section{Quantum Games}

Any quantum system which can be manipulated by two or more parties, and where some utility of the moves can be reasonably defined, may be conceived as a quantum game [8]-[10]. For example, a two-player quantum game $\Gamma=\left(\mathcal{H}, \rho, P_{A}, P_{B}\right)$ is completely specified by the underlying Hilbert

space $\mathcal{H}$ of the physical system, the initial state $\rho \in \mathcal{S}(\mathcal{H})$, where $\mathcal{S}(\mathcal{H})$ is the associated state space and $\rho=\rho_{A} \otimes \rho_{B}$ describes the players, say Alice (A) and Bob (B), initial strategies $\rho_{A}$ and $\rho_{B}$. The pay-off (utility) 
functions $P_{A}$ and $P_{B}$ specify the pay-off for each player. Quantum tactics $S_{A}$ and $S_{B}$ are linear (quantum) operations, that is, a completely positive tracepreserving map mapping the state space on itself. Employing a tactics, that is performing the appropriate linear map, describes a change of the players strategy. The quantum game's definition may also include certain additional rules, such as the order of the implementation of the respective quantum strategies. We also exclude the alteration of the pay-off during the game. The generalization for the N players case is obvious. Schematically we have:

$$
\rho \stackrel{\left(S_{A}, S_{B}\right)}{\longmapsto} \sigma \Rightarrow\left(P_{A}, P_{B}\right) .
$$

\section{Quantum Market Games}

It is tempting to check if quantum game theory may be suitable for description of market transactions. A quantum game like description of market phenomena in terms of supply and demand curves was proposed in Ref. [11]-[13]. In this approach quantum strategies are vectors in some Hilbert space and can be interpreted as superpositions of trading decisions. For an economist (or trader) they form the potential "quantum board". Strategies and not the apparatus nor the installation for actual playing are at the very core of the theory. If necessary the actual subject of investigation may consist of single traders, teams of traders or even the whole market. Due to the possible economics context the quantum strategies reveal a lot of interest-

ing properties. Supply strategies of market objects are Fourier transforms of their respective demand states [13]. 
Of course, sophisticated equipment built according to quantum rules may be necessary for generating or clearing quantum market but we must not exclude the possibility that human consciousness (brain) performs that task equally well. Even more, a sort of quantum playing board may be the natural theater of "conflict games" played by our consciousness. The agents (market players) strategies are described in terms state vectors $|\psi\rangle$ belonging to some Hilbert space $\mathcal{H}[10,12]$. The probability densities of revealing the agents, say Alice and Bob, intentions are described in terms of random variables $p$ and $q$ :

$$
\frac{\left|\langle q \mid \psi\rangle_{A}\right|^{2}}{{ }_{A}\langle\psi \mid \psi\rangle_{A}} \frac{\left|\langle p \mid \psi\rangle_{B}\right|^{2}}{{ }_{B}\langle\psi \mid \psi\rangle_{B}} d q d p,
$$

where $\langle q \mid \psi\rangle_{A}$ is the probability amplitude of offering the price $q$ by Alice who wants to buy and the demand component of her state is given by $|\psi\rangle_{A} \in$ $\mathcal{H}_{A}$. Bob's amplitude $\langle p \mid \psi\rangle_{B}$ is interpreted in an analogous way (opposite position). A short look at error theory (second moments of a random variable describe errors), Markowitz's portfolio theory and L. Bachelier's theory of options (the random variable $q^{2}+p^{2}$ measures joint risk for a stock buyingselling transaction) suggest the following definition of the risk inclination operator (a quantum observable):

$$
H\left(\mathcal{P}_{k}, \mathcal{Q}_{k}\right):=\frac{\left(\mathcal{P}_{k}-p_{k 0}\right)^{2}}{2 m}+\frac{m \omega^{2}\left(\mathcal{Q}_{k}-q_{k 0}\right)^{2}}{2},
$$

where $p_{k 0}:=\frac{{ }_{k}\left\langle\psi\left|\mathcal{P}_{k}\right| \psi\right\rangle_{k}}{{ }_{k}\langle\psi \mid \psi\rangle_{k}}, q_{k 0}:=\frac{{ }_{k}\left\langle\psi\left|\mathcal{Q}_{k}\right| \psi\right\rangle_{k}}{{ }_{k}\langle\psi \mid \psi\rangle_{k}}, \omega:=\frac{2 \pi}{\theta} . \theta$ denotes the characteristic time of transaction [12] which is, roughly speaking, an average time spread between two opposite moves of a player (e. g. buying and selling the same asset). The parameter $m>0$ measures the risk asymmetry between 
buying and selling positions.

Analogies with quantum harmonic oscillator allow for the following characterization of quantum market games. The constant $h_{E}$ describes the minimal inclination of the player to risk. It is equal to the product of the lowest eigenvalue of $H\left(\mathcal{P}_{k}, \mathcal{Q}_{k}\right)$ and $2 \theta .2 \theta$ is in fact the minimal interval during which it makes sense to measure the profit [11].

Except the ground state all the strategies $H\left(\mathcal{P}_{k}, \mathcal{Q}_{k}\right)|\psi\rangle=$ const $|\psi\rangle$ are giffens that is goods that do not obey the law of demand and supply, see bellow. It should be noted here that in a general case the operators $\mathcal{Q}_{k}$ do not commute because traders observe moves of other players and often act accordingly. One big bid can influence the market at least in a limited time spread. Therefore it is natural to apply the formalism of noncommutative quantum mechanics where one considers

$$
\left[x^{k}, x^{l}\right]=i \Theta^{k l}:=i \Theta \epsilon^{k l}
$$

The analysis of harmonic oscillator in more then one dimensions imply that the parameter $\Theta$ modifies the constant $\hbar_{E} \rightarrow \sqrt{\hbar_{E}^{2}+\Theta^{2}}$ and, accordingly, the eigenvalues of $H\left(\mathcal{P}_{k}, \mathcal{Q}_{k}\right)$. This has the natural interpretation that moves performed by other players can diminish or increase one's inclination to taking risk. 


\section{Market as a measuring apparatus}

When a game allows a great number of players in then it is useful to consider it as a two-players game: the trader $|\psi\rangle_{k}$ whom we are observing against the Rest of the World (RW). The concrete algorithm $\mathcal{A}$ that is used for clearing the market may allow for an effective strategy of RW (for a sufficiently large number of players the single player strategy should not influence the form of the RW strategy). If one considers the RW strategy it make sense to declare its simultaneous demand and supply states because for one player RW is a buyer and for another it is a seller.

To describe such situations it is convenient to use the Wigner formalism. The pseudo-probability $W(p, q) d p d q$ on the phase space $\{(p, q)\}$ known as the Wigner function is given by

$$
\begin{aligned}
W(p, q) & :=h_{E}^{-1} \int_{-\infty}^{\infty} e^{i \hbar_{E}^{-1} p x} \frac{\left\langle q+\frac{x}{2} \mid \psi\right\rangle\left\langle\psi \mid q-\frac{x}{2}\right\rangle}{\langle\psi \mid \psi\rangle} d x \\
& =h_{E}^{-2} \int_{-\infty}^{\infty} e^{i \hbar_{E}^{-1} q x} \frac{\left\langle p+\frac{x}{2} \mid \psi\right\rangle\left\langle\psi \mid p-\frac{x}{2}\right\rangle}{\langle\psi \mid \psi\rangle} d x
\end{aligned}
$$

where the positive constant $h_{E}=2 \pi \hbar_{E}$ is the dimensionless economic counterpart of the Planck constant. Recall that this measure is not positive definite except for very special cases. In a general case the pseudo-probability density of RW is a countable linear combination of appropriate Wigner functions, $\rho(p, q)=\sum_{n} w_{n} W_{n}(p, q), w_{n} \geq 0, \sum_{n} w_{n}=1$. The diagrams of the integrals of the RW pseudo-probabilities

$$
F_{d}(\ln c):=\int_{-\infty}^{\ln c} \rho(p=\text { const. }, q) d q
$$


(RW bids selling at $\exp (-p)$ )

and

$$
F_{s}(\ln c):=\int_{-\infty}^{\ln \frac{1}{c}} \rho(p, q=\text { const. }) d p
$$

( RW bids buying at $\exp q$ ) against the argument $\ln c$ may be interpreted as the dominant supply and demand curves in Cournot (French) convention, respectively [13]. Note, that due to the lack of positive definiteness of $\rho, F_{d}$ and $F_{s}$ may not be monotonic functions. Textbooks on economics give examples of such departures from the low of supply and demand (Giffen paradox). Fashion business and work supply are the source of everyday examples of such assets.

\section{Giffen paradoxes}

Note that the asymmetric crater-like hollow in (Figure 1) has the minimum bellow zero, the fact which qualitatively distinguishes the Wigner function from the supply and demand distributions for models formulated in the realm of the classical probability theory in which the measure of the probability has to be nonnegative.

The intersection of the surface of the diagram with the surface given by $p=$ constant represents the conditional probability density which is the measure of the probability for the withdrawal price of the player in the situations when this price is constant during the act of selling. The withdrawal price is defined as the maximal (minimal) price the player is going to pay (obtain) 


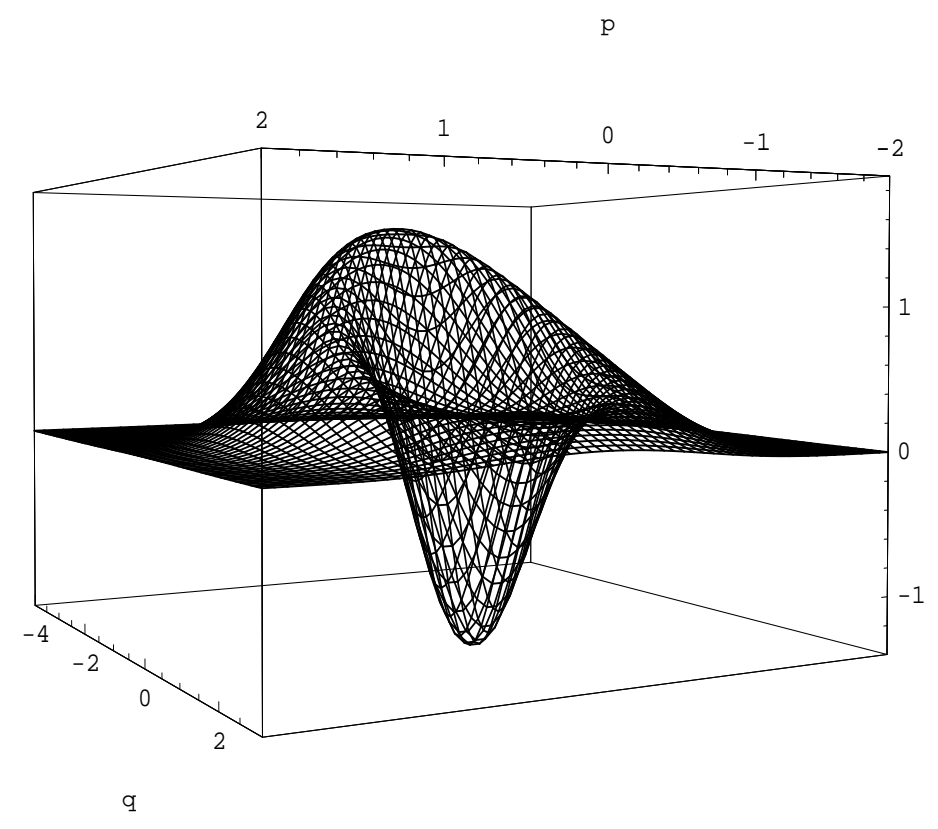

Figure 1: Exemplary plot of a Wigner function .

for the asset in question [14, 15, 13].

The cross sections for the negative values of the Wigner function are characteristic for the situation of a giffen strategy. The suitable integrals for these curves represent fully rational situations for which the demand (or supply) cease to be a monotonous function. The example of such a reaction of the player (it might be the rest of the world) is illustrated in Figure 2. We observe here the lack of the property of the monotonicity for the demand (or supply) curves (Giffen paradox). In this context it is worthy to raise the question whether the legendary captain Giffen, after observing a market anomaly which is contradictory to the law of demand, has recorded 


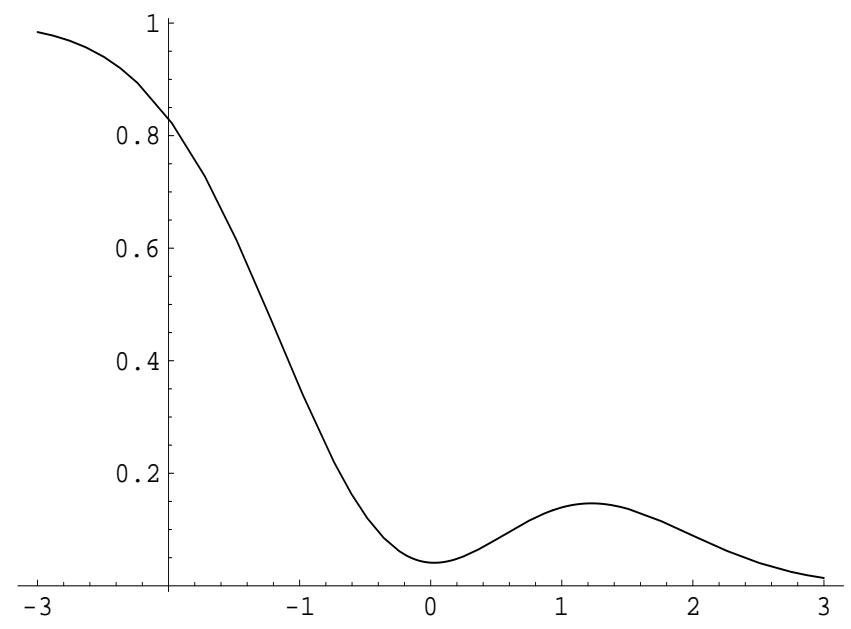

Figure 2: Non-monotonous conditional demand: the integral curve for the intersection of the surface from Figure 1 with the plain $p=0.4\left[\frac{\hbar_{E}}{\sigma}\right]$ ).

the surprising (although having logical explanation) demand that decreases after the fall of the price, or simply noticed the destructive interference which had been the effect of a careful demand transformation characteristic for a intelligent (hence acting rationally) but poor consumer [16]. The authors incline towards the second answer. It has the advantage of being capable of falsification which is a consequence of the precision qualitative predictions for this phenomenon made by the quantum theory.

Therefore it seems important to look after the conditions of the market under which the strategies described by normal distributions do not lead to the maximization of value of the intensity of the gain ${ }^{1}$ [14]. They might explain the circumstances in which we met the Giffen paradoxes.

\footnotetext{
${ }^{1}$ see also E. W. Piotrowski's lecture in current issue
} 


\section{$6 \quad$ Summary and outlook}

All this tempt us into formulating the quantum anthropic principle of the following form. At earlier civilization stages markets are governed by classical laws (as classical logic prevailed in reasoning) but the incomparable efficacy of quantum algorithms in multiplying profits will result in continuous change in human attitude towards quantum information processing. The growing significance of quantum phenomena in modern technologies and their influence on economics will result in quantum behaviour prevailing over the classical one. Therefore we envisage markets cleared by quantum algorithms (computers), quantum auctions providing agents with new means [15] and quantum games being important tools in social sciences, economics and biology [15]-[21].

\section{References}

[1] P. W. Shor, "Algorithms for quantum computation: discrete logarithms and factoring", in S. Goldwasser, ed., Proceedings of the 35th Symposium on Foundations of Computer Science, Santa Fe, NM, 20-22 November 1994 (Los Alamitos, CA: IEEE Computer Society Press 1994) 124-134.

[2] D. R. Simon, "On the power of quantum computation", in S. Goldwasser, ed., Proceedings of the 35th Symposium on Foundations of Computer Science, Santa Fe, NM, 20-22 November 1994 (Los Alamitos, CA: IEEE Computer Society Press 1994) 116-123. 
[3] C. H. Bennett and G. Brassard, "Quantum cryptography: Public-key distribution and coin tossing", in Proceedings of the IEEE International Conference on Computers, Systems and Signal Processing, Bangalore, India, December 1984 (New York: IEEE 1984) 175-179

[4] A. Ekert, "Quantum cryptography based on Bell's theorem, Phys. Rev. Lett. 67 (1991) 661-663.

[5] R. Cleve and H. Buhrman, "Substituting quantum entanglement for communication", Phys. Rev. A56 (1997) 1201-1204; W. van Dam, P. Høyer and A. Tapp, "Multiparty quantum communication complexity", PHYS REV A 60 (1999) 2737; quant-ph/9710054.

[6] J. Milnor, Games against nature, in R. M. Thrall, C. H. Coombs and R. L. Davis, eds., Decision Processes (New York: John Wiley \& Sons 1954) 49-59.

[7] J. von Neumann and O. Morgenstern, "Theory of Games and Economic Behavior", (Princeton: Princeton University Press 1953).

[8] E. Farhi and S. Gutmann, "Quantum computation and decision trees", MIT preprint CTP-2651 (1997), quant-ph/9706062.

[9] D. Meyer, "Quantum strategies", Phys. Rev. Lett. 82 (1999) 1052.

[10] A. P. Flitney, D. Abbott, "An introduction to quantum game theory"; quant-ph/0208069. 
[11] E. W. Piotrowski and J. Sładkowski, "Quantum-like approach to financial risk: quantum anthropic principle", Acta Phys. Pol. B32 (2001) 3873.

[12] E. W. Piotrowski, J. Sładkowski, "Quantum Bargaining Games", Physica A 308 (2002) 391-401.

[13] E. W. Piotrowski, J. Sładkowski, "Quantum Market Games", Physica A 312 (2002) 208.

[14] E. W. Piotrowski, J. Sładkowski, "The Merchandising Mathematician Model", Physica A in press.

[15] E. W. Piotrowski, J. Sładkowski, "Quantum English Auctions", Physica A in press.

[16] E. W. Piotrowski, J. Sładkowski and J. Syska, "Interference of quantum strategies", Physica A in press; quant-ph/0205087.

[17] E. W. Piotrowski, "Spin Systems in Contact with Thermostat", Physica A 121 (1983) 135.

[18] J. Łuczka, M. Niemiec, E. W. Piotrowski, "Randomly Interrupted Diffusion, Phys. Lett. A 167 (1992) 475.

[19] A. Iqbal, A. H. Toor, "Evolutionary stable strategies in quantum games", Phys. Lett. A 280 (2001) 249.

[20] I. Bednarek, R. Mańka, Int. J. Mod. Phys. D 10 (2001) 607. 
[21] S. Waite, "Quantum investing", (London: Texere Publishing 2002). 